\title{
Effects of submental stimulation for several consecutive nights in patients with obstructive sleep apnoea
}

Wataru Hida, Shinichi Okabe, Hiroshi Miki, Yoshihiro Kikuchi, Osamu Taguchi, Tamotsu Takishima, Kunio Shirato

\begin{abstract}
Background - It has previously been reported that short term submental stimulation can reduce the frequency of apnoea and improve sleep architecture in patients with obstructive sleep apnoea. The effects of submental stimulation during consecutive nights on apnoea or on daytime sleepiness have not, however, been studied.
\end{abstract}

Methods - Patients with obstructive sleep apnoea were studied by polysomnography on a control night, for five consecutive nights of submental stimulation, and on three following nights $(n=8)$. A multiple sleep latency test $(M S L T)(n=8)$ and measurement of the upper airway resistance $(n=5)$ were performed during the day after the polysomnographic study, on the control night, and on the fifth stimulation night. In an additional five patients with obstructive sleep apnoea, matched for age, sex, and weight, the effects of two nights of stimulation were examined for comparison. Submental stimulation began when an apnoea lasted for five seconds and stopped with the resumption of breathing as detected by oronasal flow.

Results - The apnoea index, the number of times per hour that $\mathrm{SaO}_{2}$ dropped below $85 \%$ ( $\mathrm{SaO}_{2}<85 \%$ /hour), and the total apnoea duration expressed as a percentage of total sleep time during stimulation nights decreased to approximately $\mathbf{5 0 \%}$ of the corresponding values on the control night. This improvement persisted for at least two nights after the five consecutive stimulation nights, but not after the two consecutive stimulation nights. Sleep architecture and MSLT following the stimulation nights improved but upper airway resistance did not change. Conclusions - Submental stimulation for five consecutive nights in patients with obstructive sleep apnoea improved the breathing disturbance, sleep quality, and daytime sleepiness. The effect lasted for the following two nights, but did not completely abolish the sleep disordered breathing.

(Thorax 1994;49:446-452)

An imbalance in the activity of upper airway dilating muscles and the inspiratory pump muscles leads to upper airway obstruction in patients with obstructive sleep apnoea. ${ }^{12}$ Conversely, an increase in the upper airway muscle activity which overcomes the intraluminal negative pressure generated by the contraction of inspiratory pump muscles may open an obstructed upper airway. In animal experiments Gottfried and coworkers ${ }^{3}$ reported that electrical stimulation of hyoid muscles reduced upper airway resistance, and a significant decrease in inspiratory upper airway resistance during electrical stimulation of the genioglossus has also been reported. ${ }^{4}$ Based on these animal experiments we have used percutaneous electrical stimulation in the submental region during apnoea in patients with obstructive sleep apnoea and found a reduction in the frequency of apnoeic episodes and an improvement in the sleep architecture. ${ }^{56}$ In these previous studies submental stimulation has been performed on only one or two nights, ${ }^{56}$ but the effects of submental stimulation over several consecutive nights on apnoeic episodes and daytime function have not been studied. In this study we have examined the effects of submental stimulation on five consecutive nights and during the nights following these stimulation nights. Furthermore, we have performed multiple sleep latency testing $(\mathrm{MSLT})^{7}$ to estimate the improvement of excessive daytime sleepiness, and have measured upper airway resistance to assess whether submental stimulation changes the dimensions of the upper airway during the daytime.

\section{Methods}

SUBJECTS

Thirteen men with obstructive sleep apnoea previously diagnosed by polysomnography were studied. All were habitual snorers and 10 complained of excessive daytime sleepiness and slight morning headache. All were clinically stable and free from lung disease, heart failure, or clearly definable upper airway abnormalities. They were divided into two groups, one receiving submental stimulation for five nights and the other for only two nights. The anthropometric data, spirometric measurements, blood gas tensions, and haemoglobin concentrations for the two groups are shown in table 1. Four patients were obese with body mass indices over $30 \mathrm{~kg} / \mathrm{m}^{2}$; three were in the group stimulated for five nights and one was in the group stimulated for two nights. Vital capacity (VC) and forced expira- 
Table 1 Mean (SE) anthropometric data, basal pulmonary function, and haemoglobin concentration of patients with obstructive sleep apnoea

\begin{tabular}{lcc}
\hline Variable & $\begin{array}{c}\text { Five nights of } \\
\text { stimulation } \\
(n=8)\end{array}$ & $\begin{array}{c}\text { Two nights of } \\
\text { stimulation } \\
(n=5)\end{array}$ \\
\hline Age (years) & $51 \cdot 4(3 \cdot 2)$ & $53 \cdot 8(7 \cdot 3)$ \\
Height $(\mathrm{cm})$ & $165 \cdot 1(1 \cdot 2)$ & $165 \cdot 2(2 \cdot 8)$ \\
Weight $(\mathrm{kg})$ & $76 \cdot 8(4 \cdot 1)$ & $73 \cdot 6(5 \cdot 2)$ \\
Body mass index $\left(\mathrm{kg} / \mathrm{m}^{2}\right)$ & $28 \cdot 1(1 \cdot 3)$ & $26 \cdot 8(2 \cdot 8)$ \\
Submental skin thickness (cm) & $0 \cdot 9(0 \cdot 1)$ & $0 \cdot 8(0 \cdot 1)$ \\
$\%$ VC (\% predicted) & $100 \cdot 6(5 \cdot 7)$ & $109 \cdot 8(3 \cdot 3)$ \\
FEV $/ \mathrm{VC}(\%)$ & $77 \cdot 7(3 \cdot 0)$ & $82 \cdot 7(2 \cdot 4)$ \\
pH & $7 \cdot 39(0 \cdot 01)$ & $7 \cdot 39(0 \cdot 01)$ \\
$\mathrm{PaO}_{2}(\mathrm{kPa})$ & $10 \cdot 7(0 \cdot 6)$ & $10 \cdot 4(0 \cdot 4)$ \\
$\mathrm{PaCO}_{2}(\mathrm{kPa})$ & $5 \cdot 4(0 \cdot 1)$ & $5 \cdot 3(0 \cdot 1)$ \\
$\mathrm{Haemoglobin}$ concentration $(\mathrm{g} / \mathrm{dl})$ & $14 \cdot 9(0 \cdot 6)$ & $15 \cdot 5(0 \cdot 6)$ \\
\hline
\end{tabular}

$\mathrm{VC}=$ slow vital capacity; $\mathrm{FEV}_{1}=$ forced expiratory volume in one second $\mathrm{PaO}_{2}=$ arterial oxygen pressure; $\mathrm{PaCO}_{2}=$ arterial carbon dioxide pressure.

tory volume in one second $\left(\mathrm{FEV}_{1}\right)$ were measured with a 13.5 litre spirometer (Tatebe Seishudo Co, Tokyo, Japan). The predicted normal values of Cotes $^{8}$ were used. Arterial blood gas tensions and $\mathrm{pH}$ were measured with a $\mathrm{pH} /$ blood gas analyser (Model 213; Instrumentation Laboratories, Lexington, Massachusetts, USA). There were no significant differences in these variables between the two groups. Skin thickness measured by calipers in the submental area did not differ between the groups. Each patient gave informed consent to the protocol which was approved by the Human Research Committee of our institution.

\section{SLEEP STUDIES}

Overnight sleep studies were performed in a quiet darkened room using standard polysomnographic techniques including electroencephalography (EEG), electro-oculography (EOG), and submental electromyography (EMG) with surface electrodes to determine sleep stages. ${ }^{9}$ Airflow at the nose and mouth was recorded with two thermistors and respiratory effort was assessed by inductive plethysmography (Respitrace; Ambulatory Monitoring, Ardsley, New York, USA) at the level of the mid thorax and umbilicus. Arterial oxygen saturation $\left(\mathrm{SaO}_{2}\right)$ was measured continuously with a pulse oximeter (Biox 3700; Ohmeda, Boulder, Colorado, USA). Body position was monitored by a position meter. All variables were recorded continuously on a multichannel thermal polygraph (Recti-horiz-8K; NEC San-ei, Tokyo, Japan) and data recorder (A109; Sony, Tokyo, Japan).

Apnoea was defined as the cessation of airflow at the nose and mouth lasting longer than 10 seconds. ${ }^{5610}$ Obstructive apnoea was identified as an absence of airflow signals despite continuing thoracoabdominal movement, central apnoea as stopping of airflow signals with a complete absence of thoracoabdominal movements, and mixed apnoea as both central and obstructive components during the apnoeic event. ${ }^{5610}$ Hypopnoea was defined as a fall in $\mathrm{SaO}_{2}$ of $4 \%$ or more from the preceding stable $\mathrm{SaO}_{2}$ when asleep, associated with a reduction of $50 \%$ in thoracoabdominal amplitude (Respitrace sum signal). ${ }^{11}$
SUBMENTAL STIMULATION

We developed an airflow demand-type portable submental stimulator based on a plot type apparatus, as in our previous reports. ${ }^{56}$ The apparatus consists of two parts: an apnoea detection and stimulating system. It weighs approximately $300 \mathrm{~g}$ and is operated for two nights by a rechargeable 9 volt DC battery. Airflow signals were recorded by thermistors placed near the nose and mouth. The presence of apnoea was defined as any point where the intensity of the thermistors fell to less than $10 \%$ of that during tidal breathing. Two silver chloride ECG electrodes (10 $\mathrm{mm}$ in diameter) were used as stimulation electrodes, and were attached $10 \mathrm{~mm}$ apart on the skin in the proximal half submental region. Stimulation was performed with $0.2 \mathrm{~ms}$ pulses repeated at $100 \mathrm{~Hz}$ at an intensity of 5-30 volts. Electrical stimulation began when apnoea lasted for five seconds, and was stopped after resumption of breathing was detected by oronasal airflow, or after a maximum of 20 seconds. The preset stimulation intensity was determined for each patient while awake by choosing a value just below the threshold of mild pain. If the oronasal airflow did not appear within five seconds of the onset of stimulation, the intensity automatically increased stepwise to a $50 \%$ increase for the following five seconds, and to a $75 \%$ increase from the initial intensity for the next 10 seconds. The stepwise increase in stimulation intensity always returned to the initial level. The timing of stimulation was not dependent on the respiratory cycle.

\section{MULTIPLE SLEEP LATENCY TEST (MSLT)}

An MSLT was performed according to the recommendations of the American Sleep Disorders Association. ${ }^{7}$ The subjects were placed in a dark room for 20 minutes four times during the day $(10.00,12.00,14.00$, and 16.00 hours). Polysomnographic recordings were performed during these times for those who fell asleep. Sleep latency was measured when the first epoch of any stage of sleep appeared. Each sleep latency time and the mean value of four sleep latency times were obtained.

\section{UPPER AIRWAY RESISTANCE}

Inspiratory and expiratory upper airway resistances were determined by simultaneously recording respiratory airflow and the pressure difference between the mouth and the lower pharynx. Mouth flow was obtained with a pneumotachometer (Fleisch no. 2) and a differential pressure transducer (MP-45, $\pm 2 \mathrm{~cm} \mathrm{H} \mathrm{H}_{2} \mathrm{O}$; Validyne Corporation, Northridge, California, USA). The pressure difference between the mouth and the lower pharynx was obtained by a differential pressure transducer $\left(\mathrm{MP}-45, \pm 50 \mathrm{~cm} \mathrm{H}_{2} \mathrm{O}\right.$; Validyne Corporation) calibrated with a water manometer. Pharyngeal pressure was measured at the level of the epiglottis using a polyethylene catheter, $80 \mathrm{~cm}$ in length and $1.4 \mathrm{~mm}$ internal diameter, with three or four side holes in the distal $1 \mathrm{~cm}$ of the tube, and a 
plugged end. To prevent obstruction of the catheter by secretions, a continuous flow of $1 \mathrm{ml} / \mathrm{s}$ was maintained through it from an oxygen cylinder. ${ }^{1213}$ The catheter position was confirmed by laryngoscopy before and after each study. Pressure and mouth flow signals were displayed on the $\mathrm{X}$ and $\mathrm{Y}$ axes, respectively, of a storage oscilloscope (R5103N Tektronix Beaverton, Oregon, USA). The inspiratory and expiratory resistances were taken from the slope of the linear portion of the pressure-flow relation determined at zero flow at the inspiratory and expiratory phase, respectively.

\section{PROTOCOLS}

Eight men with obstructive sleep apnoea (table 1) underwent a polysomnographic study in an all night session for two nights without stimulation, five consecutive nights with stimulation, and the following three nights without stimulation. These patients performed the MSLT as previously described during the day after the control night and the fifth stimulation night. Furthermore, in five patients upper airway resistance was measured in the supine position during wakefulness following the MSLT. For comparison, an additional five patients with obstructive sleep apnoea, matched for age, sex, and weight (table 1), underwent a polysomnographic study on two nights without stimulation, on two consecutive nights with stimulation, and on the following night without stimulation. The "control night" was defined as the second night studied polysomnographically without stimulation, a "stimulation night" as a night studied using the portable demand-type stimulator, and the "night after stimulation" as the following night without stimulation.

\section{DATA ANALYSIS}

The following polysomnographic variables were measured: total sleep time (TST), apnoea index defined as apnoeic episodes per hour, hypopnoea index defined as hypopnoeic episodes per hour, the number of times per hour that $\mathrm{SaO}_{2}$ dropped below $85 \%\left(\mathrm{SaO}_{2}<85 \%\right.$ ) hour), ${ }^{514}$ total apnoea duration expressed as a percentage of TST, mean duration of apnoea, sleep onset latency (time from lights out to sleep onset), and sleep stage characteristics on the control night, stimulation nights, and nights after stimulation. Repeated polysomnographic variables were analysed by one way analysis of variance (ANOVA). ${ }^{15}$ If significance was identified by ANOVA a comparison of variables between the control night and stimulation night, or the control night and the night after stimulation, was performed using a paired two tailed Student's $t$ test. Four sleep latency tests before and after stimulation were analysed by two way ANOVA, and each sleep latency test and the MSLT before and after successive stimulation nights were compared by a paired two tailed Student's $t$ test. The upper airway resistance before and after the stimulation nights was also analysed by a paired two tailed Student's $t$ test. Statistical significance was defined as $p<0.05$. Data are expressed as mean (SE).

\section{Results}

Polysomnographic data on the control night, the fifth stimulation night, and the first night after stimulation in patients who had submental stimulation for five consecutive nights are shown in table 2. Sleep disordered breathing, assessed by apnoea index, total apnoea duration during sleep, mean duration of apnoea, and $\mathrm{SaO}_{2}<85 \%$ /hour, was improved significantly on the fifth stimulation night. The reduced total apnoea duration was the result of a significant decrease in apnoea duration during non-REM sleep but not during REM sleep. The hypopnoea index on the control night was small and was not affected by stimulation.

Figure 1 shows a part of the polysomnographic recording obtained during non-REM sleep from a patient on the fifth stimulation night. After five seconds from the onset of apnoea, which was detected by oronasal airflow, submental stimulation via the skin was started, and after four or seven seconds of submental stimulation breathing resumed, at which point stimulation was immediately stopped. Electrical stimulation did not increase the EEG frequency with the appearance of alpha waves and a simultaneous increase in the EMG amplitude after the stimulation

Table 2 Mean (SE) polysomnographic variables on control night, the fifth stimulation night, and the first night after stimulation in the group stimulated for five nights $(n=8)$

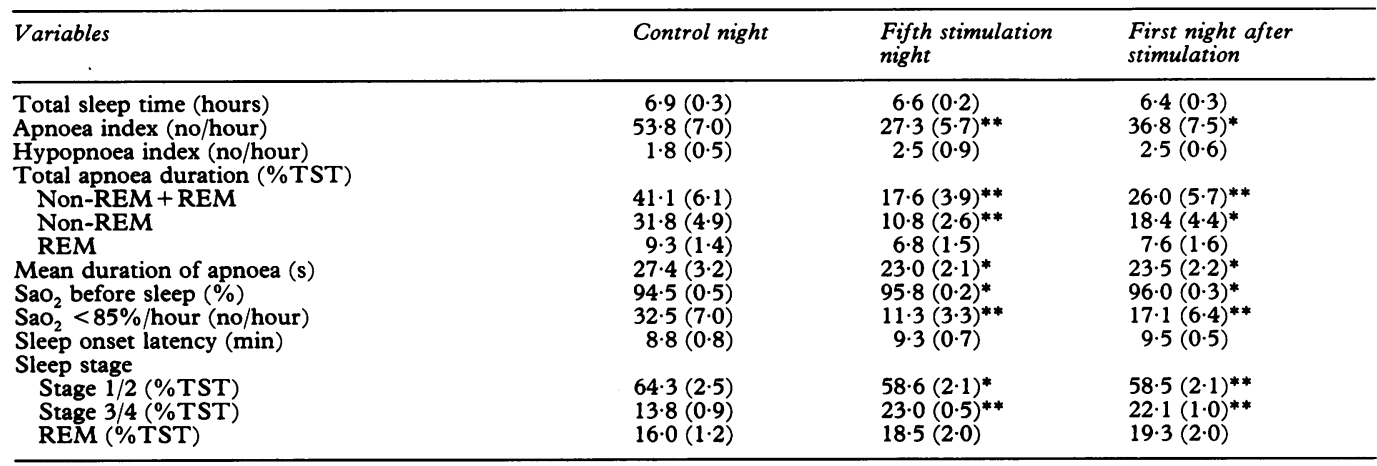

TST = total sleep time; $\mathrm{REM}=$ rapid eye movement sleep; $\mathrm{SaO}_{2}=$ arterial oxygen saturation; $\mathrm{SaO}_{2}<85 \% /$ hour $=$ number of times per hour that $\mathrm{SaO}_{2}$ dropped below $85 \%$

${ }^{*} \mathrm{p}<0.05,{ }^{* *} \mathrm{p}<0.01 \mathrm{v}$ corresponding values before stimulation night. 


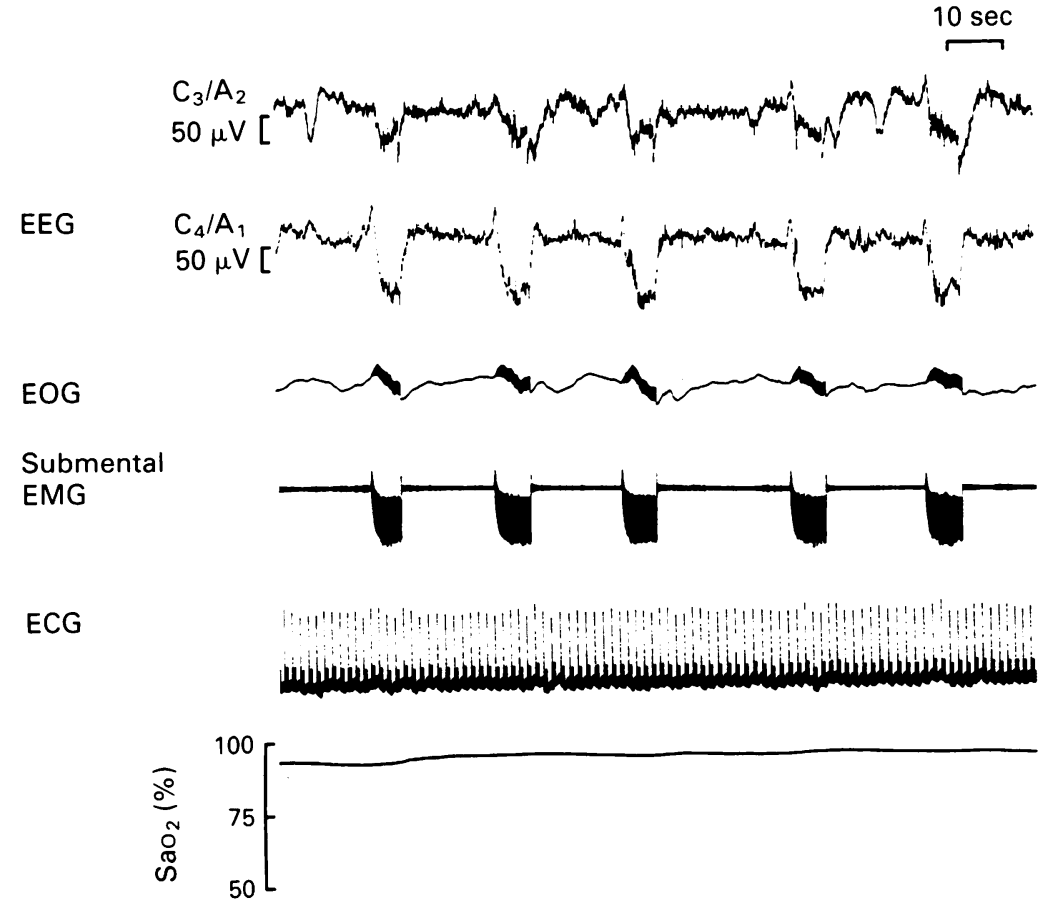

Airflow

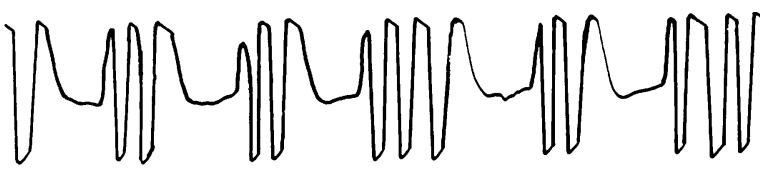

Rib cage

+ abdomen

Stimulation

Figure 1 Part of the polysomnographic recording during non-REM sleep in one patient with obstructive sleep apnoea on a night with submental stimulation. See text for further explanations. induced artifact, and seemed to resolve apnoeic episodes without definable arousal.

The apnoeic episodes on the other stimulation nights also improved as shown in fig 2 . The apnoea index, $\mathrm{SaO}_{2}<85 \% /$ hour, and total apnoea duration/TST on the first and second nights decreased significantly compared with the corresponding values on the control night. Furthermore, significant after effects of submental stimulation were found. The values of these three parameters and mean duration of apnoea were still significantly lower on the first night after stimulation (fig 2, table 2), and $\mathrm{SaO}_{2}$ $<85 \%$ /hour and total apnoea duration decreased significantly on the second night after stimulation. The decrease in total apnoea duration during the nights after stimulation was observed during non-REM sleep (table 2). On the third night after stimulation the apnoeic episodes returned to the control level. $\mathrm{SaO}_{2}$ before sleep improved on the fifth stimulation night, and also improved on the first (table 2 ) and second nights after stimulation (96.6 (0.5)\%, p < 0.05 compared with the control value). The effects of stimulation therefore lasted for at least two nights after stimulation for five successive nights.

The distribution of sleep stages and sleep onset latency for the control night, the fifth stimulation night, and the first night after stimulation are shown in table 2. Stages 1 and 2 decreased significantly whereas stages 3 and 4 increased significantly both on the fifth stimulation night and on the first night after stimulation. REM stages and sleep onset latency did not change throughout the study. None of the subjects was awakened by the stimulation and none complained of skin pain or any other discomfort the following morning. Symptoms of excessive daytime sleepiness and morning headache also improved after the stimulation nights.

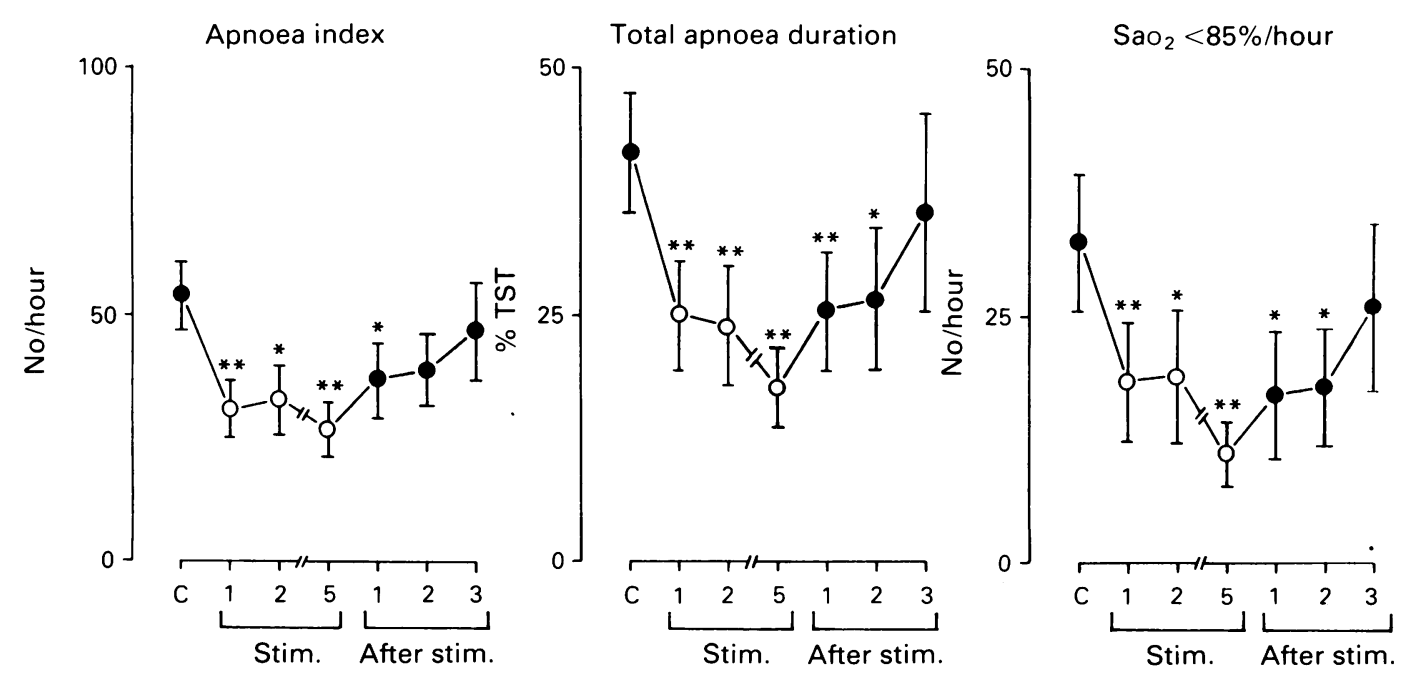

Figure 2 Apnoea index, total apnoea duration during sleep expressed as a percentage of total sleep time (TST), and $\mathrm{SaO}_{2}<85 \%$ /hour on the control night $(C)$, the first, second, and fifth nights of stimulation, and the following first, second, and third nights. The apnoea index, total apnoea duration during sleep/TST, and SaO $2<85 \% /$ hour decreased on stimulation nights compared with the values on the control night. The apnoea index was decreased on the first night after stimulation, and the other two parameters were decreased for at least two nights after stimulation compared with values on the control night. On the third night after stimulation the values of these parameters returned to the control night level. ${ }^{*} p<0.05,{ }^{* *} p<0.01 \mathrm{v}$ control night. 


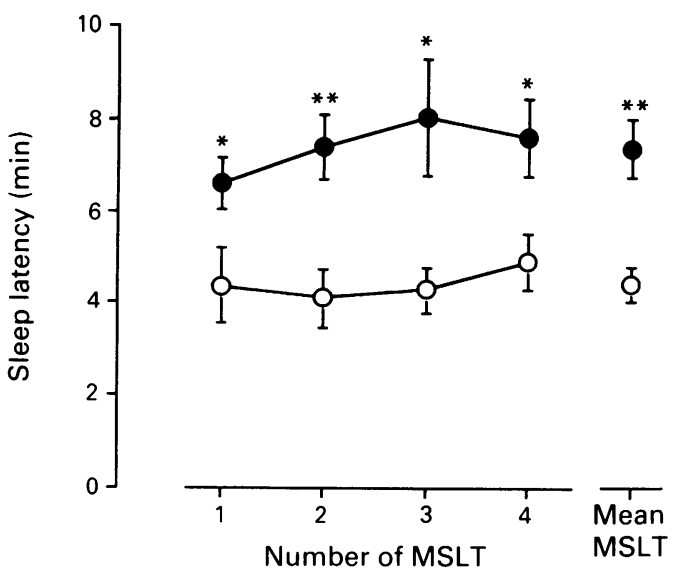

Figure 3 Mean value of each sleep latency test (MSLT) and mean of four sleep latency tests before and after five consecutive nights of stimulation. ${ }^{*} p<0.05,{ }^{* *} p<0.01 \mathrm{v}$ value before stimulation nights.

The four sleep latency times and the mean MSLT after five consecutive nights of stimulation were prolonged significantly compared with those on the control day (fig 3 ). Inspiratory and expiratory upper airway resistances before stimulation nights were $0.75(0.12)$ and 0.60 $(0 \cdot 11) \mathrm{cm} \mathrm{H}_{2} \mathrm{O} / \mathrm{l} / \mathrm{s}$, respectively, and inspiratory and expiratory upper airway resistances after five consecutive nights of stimulation were 0.71 $(0 \cdot 12)$ and $0.62(0 \cdot 10) \mathrm{cm} \mathrm{H}_{2} \mathrm{O} / 1 / \mathrm{s}$, respectively. Neither inspiratory nor expiratory upper airway resistances differed before or after successive stimulation nights.

Polysomnographic data for five patients who had stimulation for two nights are shown in table 3. Apnoea index, total apnoea duration, mean duration of apnoea, and $\mathrm{SaO}_{2}<85 \%$ / hour on the second stimulation night decreased significantly compared with the corresponding values on the control night. The hypopnoea index did not change, but $\mathrm{SaO}_{2}$ before sleep improved on the stimulation night. In this group no post-stimulation effects were observed on MSLT.

\section{Discussion}

The effects of submental stimulation for five consecutive nights on apnoeic episodes have been studied during the stimulation nights and the nights after stimulation using a portable submental stimulator in patients with obstructive sleep apnoea. A significant reduction in the duration and frequency of apnoeas was found on the stimulation nights and for at least two nights after stimulation, with an associated improvement in sleep quality and daytime sleepiness. Complete abolition of the sleep disordered breathing did not occur.

In our previous paper we used a microphone attached to the skin over the cervical trachea to detect apnoeic episodes, and the stimulating system and detecting system were completely separated. $^{56}$ If the stimulating system and detecting system were combined, the latter sometimes picks up noise caused by the electrical stimulation, resulting in a malfunction. It was difficult to separate completely the stimulation frequency and the sound frequency as the frequency bands overlapped. In the present study we have therefore used oronasal flow thermistors which require lower frequency signals $(<2 \mathrm{~Hz})$ to detect apnoeic episodes, instead of the tracheal sound recordings. The stimulator operated properly with these thermistors. Apnoeic episodes are easily affected by body position and patients with obstructive sleep apnoea tend to have more apnoeas during sleep when supine than in the lateral position. ${ }^{1617}$ Body position was supine throughout the night and hence postural effects were negligible.

The mechanisms by which submental stimulation reduced the duration and frequency of apnoeic episodes during stimulation nights are not clear. One possibility is that submental stimulation resolved apnoeic episodes by arousal. Unfortunately, in the present study we could not estimate EEG arousal ${ }^{18}$ during stimulation nights because of the stimulation-induced artifact. Definable EEG arousal after stimulation was not, however, observed as shown in fig 1. Moreover, the improvement in clinical symptoms, sleep quality and MSLT suggests that arousal frequency was not increased. An alternative explanation is that submental stimulation increased muscle tone in the genioglossus and/or geniohyoid muscles, and so decreased upper airway resistance $^{34}$ or upper airway collapsibility. ${ }^{219}$ In our previous paper submental stimulation itself did not change blood pressure or heart rate, and sham stimulation in other parts of the body (arm and leg) did not reduce apnoeic episodes. ${ }^{5}$ These findings would also support the latter hypothesis.

The effectiveness of submental stimulation may depend on six factors. The first is the stimulatory condition. We used a stimulation frequency of $100 \mathrm{~Hz}$ as, in our animal experiments, we observed a maximal decrease in

Table 3 Mean (SE) polysomnographic variables on control night, the second stimulation night, and the first night after stimulation in the group stimulated for two nights $(n=5)$

\begin{tabular}{|c|c|c|c|}
\hline Variables & Control night & $\begin{array}{l}\text { Second stimulation } \\
\text { night }\end{array}$ & $\begin{array}{l}\text { First night after } \\
\text { stimulation }\end{array}$ \\
\hline $\begin{array}{l}\text { Total sleep time (hours) } \\
\text { Apnoea index (no/hour) } \\
\text { Hypopnoea index (no/hour) } \\
\text { Total apnoea duration (\%TST) } \\
\text { Mean duration of apnoea (s) } \\
\mathrm{SaO}_{2} \text { before sleep (\%) } \\
\mathrm{SaO}_{2}<85 \% / \text { hour (no/hour) }\end{array}$ & $\begin{array}{l}7 \cdot 0(0 \cdot 3) \\
29 \cdot 7(5 \cdot 8) \\
2 \cdot 5(1 \cdot 2) \\
29 \cdot 3(10 \cdot 5) \\
32 \cdot 0(8 \cdot 1) \\
95 \cdot 0(0 \cdot 3) \\
14 \cdot 4(7 \cdot 2)\end{array}$ & $\begin{array}{l}6 \cdot 6(0 \cdot 4) \\
20 \cdot 6(7 \cdot 8)^{*} \\
1 \cdot 2(0 \cdot 2) \\
13 \cdot 2(5 \cdot 8)^{*} \\
20 \cdot 5(4 \cdot 7)^{*} \\
96 \cdot 0(0 \cdot 5)^{*} \\
8 \cdot 8(7 \cdot 6)^{*}\end{array}$ & $\begin{array}{l}7 \cdot 1(0 \cdot 4) \\
27 \cdot 1(7 \cdot 0) \\
1 \cdot 3(0 \cdot 5) \\
26 \cdot 9(10 \cdot 7) \\
30 \cdot 4(8 \cdot 3) \\
95 \cdot 6(0 \cdot 6) \\
15 \cdot 7(8 \cdot 5)\end{array}$ \\
\hline
\end{tabular}

TST = total sleep time; $\mathrm{SaO}_{2}=$ arterial oxygen saturation; $\mathrm{SaO}_{2}<85 \%$ /hour = number of times per hour that $\mathrm{SaO}_{2}$ dropped below

${ }^{8} \mathrm{p}<0.05 v$ corresponding values before stimulation night. 
upper airway resistance of $>50 \mathrm{~Hz}^{4}$ The intensity of stimulation for each patient was set below the pain threshold while awake. The frequency of apnoeic episodes during stimulation nights at this intensity still remained at approximately $50 \%$ of the control nights, possibly because the intensity of genioglossus stimulation was insufficient to open the upper airway. If we had used a higher intensity stimulus this would have induced frequent arousal and led to sleep fragmentation.

The second factor is the sleep stage. The stimulation threshold used in this study was only effective on apnoeic episodes during nonREM sleep and not on those during REM sleep. It is well known that activity of the genioglossus muscle during the REM sleep apnoeic phase almost ceases, but genioglossus muscle activity during non-REM sleep apnoeic phase only decreases. We therefore suggest that the stimulation threshold required to open the upper airway during REM sleep apnoeas is greater than for non-REM apnoeas. Even in non-REM sleep, apnoeic episodes were not resolved completely. This may be because different thresholds are required for opening the upper airway in each apnoea in the different sleep stages of non-REM sleep.

The third factor is the type of apnoea. Submental stimulation is effective in abolishing obstructive apnoeas, less effective on the mixed type, and ineffective on the central type. ${ }^{5}$

The fourth factor on which the effectiveness of submental stimulation may depend is the skin thickness in the submental area. Submental skin thickness in the four obese patients $(1.0-1.5 \mathrm{~cm})$ tended to be thicker than in nonobese patients $(0 \cdot 6-0.8 \mathrm{~cm})$. Although the obese patients did not differ significantly in their response to electrical stimulation from the non-obese patients, the intensity of stimulation set while awake was greater in the former.

Another factor which may be important in the effectiveness of submental stimulation is the adhesive force in the pharynx during upper airway obstruction. If the adhesive force is strong it would become hard to open the upper airway, as shown in our previous animal experiment, ${ }^{20}$ in which case a higher intensity of stimulation may be required to open the obstructed upper airway.

Finally, the anatomical abnormality of the upper airway may affect submental stimulation, since we have failed to show any benefit from this method of submental stimulation in patients with obstructive sleep apnoea who had hypertrophy of the tonsils. ${ }^{6}$

Submental stimulation also prolonged the MSLT, although it was shorter than the normal range (that is, $>10$ minutes). ${ }^{21}$ This change of MSLT was similar to the result seen with nasal CPAP therapy. ${ }^{22} 23$ The mechanism of prolongation of the MSLT in the present study is not clear, but may be related to an improvement in sleep fragmentation, sleep deprivation, nocturnal hypoxia, or hypercapnia.

The frequency and duration of apnoeic epi- sodes were reduced on the nights after stimulation, and this effect lasted for at least two days. This may be because of an improvement in the ventilatory response to hypercapnia, sleeprelated respiration, oxygenation, sleep fragmentation, and alertness as observed in patients with obstructive sleep apnoea with nasal CPAP therapy, ${ }^{22-24}$ producing a decrease in apnoeic episodes on the nights after stimulation. A second possibility is that the upper airway submucosal oedema induced before treatment may disappear during the stimulation nights and nights after stimulation as occurs with nasal CPAP. ${ }^{25}$ Since the measurement of upper airway resistance may be less sensitive for detecting subtle degrees of airway oedema than the magnetic resonance imaging method used by Ryan et al, ${ }^{25}$ significant differences in upper airway resistance before and after the stimulation nights might not have been found. An alternative explanation is that a conditioning effect formed in the brain during the stimulation nights may reduce the apnoeic episodes during the nights after stimulation, as several investigators have argued that the conditioning hypothesis is not only relevant to normal respiratory functions ${ }^{26}$ but also to respiratory dysfunctions such as the anticipatory change in breathing pattern before muscular exercise, ${ }^{27}$ asthmatic attacks, ${ }^{28}$ and dyspnoea. ${ }^{29}$

In summary, we have observed that submental stimulation for several successive nights in patients with obstructive sleep apnoea reduced the frequency and duration of apnoeic episodes with an associated improvement in the sleep quality and daytime sleepiness. These effects remained for at least two nights following the five successive stimulation nights. However, such improvement was not complete and further studies on the usefulness of submental stimulation in the management of obstructive sleep apnoea are required.

The authors wish to thank Drs H Kurosawa, J Midorikawa and $\mathrm{T}$ Chonan for their technical assistance and Mr B Bell for reading the manuscript.

1 Brouillette RT, Thach BT. A neuromuscular mechanism maintaining extrathoracic airway patency. F Appl Physiol 1979;46:772-9.

2 Remmers JE, deGroot WJ, Sauerland EK, Anch AM Pathogenesis of upper airway occlusion during sleep. $\dot{f}$ Appl Physiol 1978;44:931-8.

3 Gottfried SB, Strohl KP, Van de Graaff W, Fouke JM, DiMarco AF. Effects of phrenic stimulation on upper airway resistance in anesthetized dogs. $\mathcal{J}$ Appl Physio 1983;55:419-26.

4 Miki H, Hida W, Shindoh C, Kikuchi Y, Chonan T, Taguchi $\mathrm{O}$, et al. Effects of electrical stimulation of the genioglossus on upper airway resistance in anesthetized dogs. Am Rev Respir Dis 1989;140:1279-84.

5 Miki H, Hida W, Chonan T, Kikuchi Y, Takishima T. Effects of submental electrical stimulation during sleep on upper airway patency in patients with obstructive sleep apnea. Am Rev Respir Dis 1989;140:1285-9.

6 Hida W, Miki H, Kikuchi Y, Chonan T, Takishima T Treatment of obstructive sleep apnea with airflow demand-type submental stimulator. In: Takishima T, Cherniack NS, eds. Control of breathing and dyspnea. Advances in the biosciences, Vol 79. Oxford: Pergamon Press,

7 Carskadon MA, Dement WC, Mitler MM, Roth T, Westbrook PR, Keenan S. Guidelines for the multiple sleep latency test (MSLT): a standard measure of sleepiness. Sleep 1986;9:519-24.

8 Cotes JE. Lung function. 4th ed. Oxford: Blackwell Scientific Publications, 1979.

9 Rechtschaffen A, Kales A. A manual of standardized terminology, techniques and scoring system for sleep stages of human subjects. Publication no. 204. Bethesda: Nationa Institutes of Health, 1968.

10 Guilleminault C, Cummiskey J, Dement WC. Sleep apnea 
syndrome; recent advances. In: Stollerman GH, ed. $A d-$ syndrome; recent advances. In: Stollerman GH, ed. Ad-
vances in internal medicine. Vol 26. Chicago: Year Book vances in internal medicine. Vol 26.

11 Gould GA, Whyte KF, Rhind GB, Airlie MAA, Catterall $\mathrm{JR}$, Shapiro CM, et al. The sleep hypopnea syndrome. Am Rev Respir Dis 1988;137:895-8.

12 Hudgel DW. Variable site of airway narrowing among obstructive sleep apnea patients. $f$ Appl Physiol 1986;61:1403-9.

13 Series F, Cormier Y, Desmeules M, Forge J La. Influence of respiratory drive on upper airway resistance in normal men. 7 Appl Physiol 1989;66:1242-9.

14 Roehrs T, Conway W, Wittig R, Zorick F, Sicklesteel J, Roth $\mathrm{T}$. Sleep-wake complaints in patients with sleeprelated respiratory disturbances. Am Rev Respir Dis 1985;132:520-3.

15 Snedecor GW, Cochran WG. Statistical methods. 7th ed. Ames: Iowa State University Press, 1980.

16 Mcevoy RD, Sharp DJ, Thornton AT. The effects of posture on obstructive sleep apnea. Am Rev Respir Dis posture on obst

17 Miki H, Hida W, Kikuchi Y, Takishima T. Effect of sleep position on obstructive sleep apnea. Tohoku f Exp Med 1988;156(Suppl):143-9.

18 EEG arousals: scoring rules and examples. A preliminary report from the sleep disorders atlas task force of the American Sleep Disorders Association. Sleep 1992;15:174-84.

19 Brouillette R, Thach B. A neuromuscular mechanism maintaining extrathoracic airway patency. $\mathcal{F}$ Appl Physiol 1979:46:772-9.

20 Miki H, Hida W, Kikuchi Y, Chonan T, Satoh M, Iwase N, et al. Effects of pharyngeal lubrication on the opening of obstructive upper airway. $f$ Appl Physiol 1992;72:2311-6. 21 Richardson GS, Carskadon MA, Flagg W, van den Hoed J, Dement WC, Mitler MM. Excessive daytime sleepiness in man: multiple sleep latency measurement in narcoleptic and control subjects. Electroencephalogr Clin Neurophysiol 1978;45:621-7.

22 Rajagopal KR, Bennett LL, Dillard TA, Tellis CJ, Tenholder MF. Overnight nasal CPAP improves hypersomnolence in sleep apnea. Chest 1986;90:172-6.

23 Berthon-Jones $M$, Sullivan CE. Time course of change in ventilatory response to $\mathrm{CO}_{2}$ with long-term therapy for ventilatory response to $\mathrm{CO}_{2}$ with long-term therapy for
obstructive sleep apnea. $\mathrm{Am}$ Rev Respir Dis 1987;135: obstructi

24 Lamphere J, Roehrs T, Wittig R, Zorick F, Conway WA Roth T. Recovery of alertness after CPAP in apnea. Chest 1989;96:1364-7.

25 Ryan CF, Lowe AA, Li D, Fleetham JA. Magnetic resonance imaging of the upper airway in obstructive sleep apnea before and after chronic nasal continuous positive airway pressure therapy. Am Rev Respir Dis airway pressure

26 Gallego J, Perruchet P. Classical conditioning of ventilatory responses in humans. $\mathcal{f}$ Appl Physiol 1991;70:676-82.

27 Tobin MJ, Perez W, Guenther SM, D'Alonzo G, Dantzke DR. Breathing pattern and metabolic behavior durin anticipation of exercise. $\mathcal{F}$ Appl Physiol 1986;60:1306-12.

28 Dekker E, Pelser HE, Groen J. Conditioning as a cause of asthmatic attack. 7 Psychosom Res 1957;2:97-108.

29 Dudley DL, Pitts-Porch AR. Psychophysiologic aspects of respiratory control. Clin Chest Med 1980;1:131-43. 